\title{
5G Network Goals: A Comparative Investigation of the Spectral Efficiencies and other Performance Parameters of 4G OFDM and 5G GFDM Signals
}

\author{
Ali Jasim Ramadhan \\ Department of Computer Techniques Engineering, College of Technical Engineering, \\ University of Alkafeel, 31003 Kufa, Province of Najaf, Republic of Iraq
}

\begin{abstract}
In this study, we present next-generation Generalized Frequency-Division Multiplexing (GFDM) based 5th-Generation $(5 \mathrm{G})$ systems which are designed to overcome the drawbacks of 4th-Generation $(4 \mathrm{G})$ systems. In $5 \mathrm{G}$ systems, the number of cyclic prefixes required is reduced by adding one to the header of each frame (subsymbols), rather than to each symbol and then transmitting the data in blocks of subcarriers and subsymbols. To meet the growing demand for reliable ubiquitous connectivity, higher energy efficiency, lower network latency and higher data rates, $5 \mathrm{G}$ communication networks will require a majo rqualitative shift. These systems have been predicted to be launched soon and many techniques have been proposed to meet the requirements of $5 \mathrm{G}$ technology. The primary techniques include new radio access techniques, green communications, device-to-device connectivity, the internet of things, big data and mobile cloud computing, network ultra-densification, massive multiple-input multiple-output, use of the millimeter-wave spectrum, network function virtualization and wireless software-defined networks. This study presents an extensive survey of these techniques. In addition, MATLAB was employed to compare the Symbol Error Rates (SERs) and spectra of GFDM (5G) and Orthogonal Frequency-Division Multiplexing (OFDM) (4G) signals, demonstrating that GFDM uses the spectrum more effectively than OFDM and yields lower SERs.
\end{abstract}

Key words: 5G, OFDM, GFDM, UFMC, FBMC, SERs

\section{INTRODUCTION}

A new generation of wireless communication systems is developed each decade. The 4th-Generation (4G) wireless communication systems, i.e., systems of the current generation were made available by Anonymous (2010). The standardization and arraying of 4th-Generation (5G) wireless systems will commence in the early 2020 's. The upgrading of the existing systems to $5 \mathrm{G}$ has attracted considerable interest following the World Radiocommunication Conference (WRC) held in 2015 which was attended by individuals associated with or a part of the global International Telecommunication Union Radiocommunication Sector (ITU-R).

According to industry professionals and instructional researchers, the $5 \mathrm{G}$ structure will provide considerably higher data rates (on the order of $10 \mathrm{Gbps}$ ) for cellular devices, despite issues such as static and irregular mobile transmission. Furthermore, Dlobal System for Mobile communications (GSM) and Enhanced Data rates for GSM Evolution (EDGE) users can avail themselves of $5 \mathrm{G}$ services at speeds $>300 \mathrm{~km} / \mathrm{h}$ owing to the extensive coverage expected from the $5 \mathrm{G} 1 \mathrm{Gbps}$ network. In addition, $5 \mathrm{G}$ systems are expected to reduce round-trip times in cellular communications. The average round-trip time is expected to decrease from $20 \mathrm{msec}$ in $4 \mathrm{G}$ systems to approximately $1 \mathrm{msec}$ in $5 \mathrm{G}$ systems. These are the current targets of $5 \mathrm{G}$ International Mobile Telecommunications (IMT) systems but they have not been confirmed, thus far.

To ensure worldwide interoperability, realize financial systems of scale, achieve ubiquitous connectivity and enable harmonization among multiple vendors, it is essential to develop a foundation for the global requirements of $5 \mathrm{G}$ systems. In general, the IMT specifications are defined by the ITU-R for each wireless technology generation. Specifically, the ITU-R defined the requirements of the previous two generations of wireless systems, namely, 4G (IMT-A) and 3rd-Generation (3G) (IMT-2000) systems. It has now commenced defining specifications for $5 \mathrm{G}$ systems. WRCs are organized every 3-4 years to review the radio regulations set by the ITU-R. The primary agenda for discussion at the next WCR is a new mobile communication spectrum.

To understand the upgrades of the existing networks in future $5 \mathrm{G}$ systems, it is necessary to review the history of wireless network advancement (Rodriguez 2015). 
Before the 1980's, an analogue voice system based on Single-Sideband (SSB) frequency modulation technology was used for wireless communication. The 1 st-Generation $(1 \mathrm{G})$ systems as they later became known were the most widely used voice service systems in the 1980 's. However, before 1966, the fabrication of digital radio structures was difficult, therefore, to obtain a system with high mobile capacity, Bell Labs developed and applied analogue technology based on Frequency-Division Multiple Access (FDMA). Thus, they established the Advanced Mobile Phone System (AMPS) a $1 \mathrm{G}$ system.

In North America, Bell Labs and related research institutes continued to research on the advancement of 2nd-Generation (2G) network systems. These systems were officially implemented in 1990 and soon, made available to the public. In particular, this upgrade involved a shift from analogue to digital systems. The GSM in Europe and Interim Standard (IS)-54 in North America were based on Time-Division Multiple Access (TDMA). Following the discontinuation of IS-54, the introduction of the Second-and-a-Half Generation $(2.5 \mathrm{G})$ technology led to the development of completely wireless communication systems in 1997. This technology provided high voice capabilities with limited data service. The US adopted a 1.25-MHz-bandwidth-based Code-Division Multiple Access (CDMA) system whiles European countries adopted General Packet Radio Service (GPRS) as an upgrade to GSM and commenced using the enhanced data rates provided by EDGE. The $2 \mathrm{G}$ wireless technology was so called because of its improvements over the previous GSM technology. In 2000, the ITU defined specifications for $3 \mathrm{G}$ systems.

The $3 \mathrm{G}$ technology included both voice and data capabilities. It was the first generation to meet international wireless communication requirements and was based on a $5 \mathrm{MHz}$ bandwidth that used Wide CDMA (WCDMA) technology. Operation was based on Frequency-Division Duplexing (FDD) and Time-Division Duplexing (TDD) approaches. Hence, the upgrade of network systems from 2-3G enhanced system data and voice services.

Subsequently, $4 \mathrm{G}$ communication systems were introduced in 2010 with the highest data rates, thus far. Technology in this generation can be classified into two types: the Worldwide Interoperability for Microwave Access (WIMAX) technology developed by the US and Long-Term Evolution (LTE) technology developed subsequently. WIMAX is Orthogonal Frequency-Division Multiplexing (OFDM) based technology that depends on Wi-Fi. The bandwidth of both WIMAX and LTE technology is $20 \mathrm{MHz}$. However, most mobile users use LTE technology. The 4G licenses required to use advanced LTE systems are auctioned in most countries. The upgrade from $3-4 \mathrm{G}$ technology led to improved data speeds that enabled video streaming on mobile devices. It has been predicted that by 2020 , the use of $5 \mathrm{G}$ technology will be approved by communication regularization authorities. As part of the $5 \mathrm{G}$ evolution, extremely high internet speeds and bandwidths with efficient energy utilization are predicted based on new layout parameters, custom design and low operating costs. It is expected that $5 \mathrm{G}$ technology will enable the creation of technological systems involving Wi-Fi networks working in synergy with mobile networks which will provide customers with continuous communication channels. Consequently, a design-model shift from single to multiple-discipline will be a core feature of the upgrade from 4-5G. Table 1 summarizes the general features of the main wireless network generations (Ramadhan, 2017).

It is necessary to provide faster data rates because of the ever-growing consumer demands for safer, smarter and faster Wi-Fi networks. The evolution of the Wi-Fi communication industry is based on efforts to increase data rates by improving system performance. In summary, such technologies have witnessed various transformations including the upgrade from analogue to digital, the change from circuit switching to packet switching and the move from cumbersome hand-held cellular phones to smart watches (Akyildiz et al., 2014a, b, 2015a-c). Despite the significant breakthroughs in communication technology, wireless communication networks require further improvement considering the drawbacks of current systems in terms of spectral resource utilization.

Evaluating overall system performance is difficult because of the restrictions posed by the available bandwidth in each generation of wireless systems. It is predicted that additional spectral allocation will be necessary in the next generation to allow network use by more subscribers at faster data rates. Furthermore, it is important to utilize the current spectrum efficiently (Ghosh et al., 2014). Ultra-dense networks and small-cell deployment are necessary to realize greater network flexibility and deliver good connectivity to subscribers at any location and time. In addition, new Wi-Fi access technologies aimed at faster data rates should be backward compatible with established solutions to ensure the highest possible quality in terms of overall network 
Table 1: Comparison of the features of different wireless generations

\begin{tabular}{|c|c|c|c|c|c|c|}
\hline $\begin{array}{l}\text { Primary features/ } \\
\text { Generation }\end{array}$ & Launch & Primary standard & System & Primary technology & Average data rate (bps) & Primary service \\
\hline $0 \mathrm{G}$ & 1946 & MTS & Analogue & FM-SSB & - & Wireless voice \\
\hline \multirow[t]{2}{*}{$0.5 \mathrm{G}$} & 1970 & ARP & Analogue & FM-SSB & - & Remote communication $1 \mathrm{G} 1 \mathrm{G}$ \\
\hline & 1980 & AMPS & Analogue & FDMA & - & Mobile communication \\
\hline \multicolumn{7}{|l|}{2 G systems } \\
\hline \multirow{2}{*}{$2 \mathrm{G}$} & 1990 & GSM & Digital & TDMA & $9.6 \mathrm{~K}$ & International communication \\
\hline & 1993 & CDMA One & Digital & CDMA & $9.6 \mathrm{~K}$ & Coded communication \\
\hline $2.5 \mathrm{G}$ & 1997 & GPRS & Digital & FDMA/ TDMA & $35-171 \mathrm{~K}$ & SMS \\
\hline $2.75 \mathrm{G}$ & 1999 & EDGE & Digital & FDMA/ TDMA & $120 \mathrm{~K}-384 \mathrm{~K}$ & MMS \\
\hline \multicolumn{7}{|l|}{3 G systems } \\
\hline $3 \mathrm{G}$ & 2000 & UMTS & Digital & WCDMA & $384 \mathrm{~K}-1 \mathrm{M}$ & Multimedia \\
\hline $3.5 \mathrm{G}$ & 2007 & HSDPA & Digital & WCDMA & $1-2 \mathrm{M}$ & Intemet \\
\hline $3.75 \mathrm{G}$ & 2009 & HSUPA & Digital & WCDMA & $2-3 \mathrm{M}$ & High data rates/Speed \\
\hline \multirow[t]{2}{*}{$4 \mathrm{G}$} & 2010 & WIMAX & Digital & OFDM & $3-10 \mathrm{M}$ & Higher data rates/Speed \\
\hline & 2013 & LTE & Digital & OFDM & $20-100 \mathrm{M}$ & Higher data rates/Speed \\
\hline $5 \mathrm{G}$ & 2020 (expected) & WwWw & Digital & GFDM (candidate) & $1-100 \mathrm{G}$ & IoT \\
\hline
\end{tabular}

performance (Boccardi et al., 2014). Therefore, we describe the current states of 10 enabling technologies that could contribute to the realization of these goals and discuss their potential roles in $5 \mathrm{G}$ wireless communication systems.

Requirements of 5G technology: Until recently, mobile phones were the only type of device that required cellular networks. However, with the proliferation of internet-based content and evices, traffic issues need to be identified and resolved to fulfil the Quality-of-Service (QOS) preconditions for different applications such as video streaming and voice over internet protocol calls. Furthermore, 5G cellular networks can support various devices including autonomous vehicles, smart watches and Internet-of-Things (IoT) devices that cannot be suitably supported by existing wireless systems (Akyildiz et al., 2016). The three primary categories of services defined by the ITU that require the support of 5G technology are massive Machine-Type Communication (MTC), mobile broadband services and ultra-reliable low-latency communication (ITU-R, 2015). Moreover, various devices and applications require complicated networks to ensure high throughput, low-latency information delivery, energy efficiency, high scalability and ubiquitous connectivity for customers. In this study, the expected objectives of $5 \mathrm{G}$ technology are discussed as a preface to understanding the potential technological means of achieving these objectives.

High data rates: Data rates have long been the primary metric for evaluating wireless communication networks. Recent years have witnessed rapid increases in the use of services such as High-Definition (HD) streaming and sharing of videos (including pervasive video and virtual reality) on mobile devices including tablets and laptops that can access wireless networks. The driving force in communication markets is the demand for higher data rates. Applications such as extremely $\mathrm{HD} 4 \mathrm{~K}$ gaming, 3D content and video streaming require data rates of approximately $25 \mathrm{Mbps}$ to ensure satisfactory user experiences. However, these rates are significantly higher than the highest available data rates currently used to support HD video streaming (Akyildiz et al., 2016).

With $5 \mathrm{G}$ networks, the peak data rates are expected to be around $10 \mathrm{Gbps}$, i.e., 100 times greater than the current $4 \mathrm{G}$ network rates (Hossain and Hasan, 2015). The rate of cell-facet record which is generally, the worst-case scenario for users in terms of data rates should be enhanced to $100 \mathrm{Mbps}$, i.e., 100 times greater than those of $4 \mathrm{G}$ networks. This rate should satisfy the worst-case scenario requirements for customers. However, intercellular interference and transmission loss make it difficult to achieve the maximum possible data rate. From the perspective of network engineering, the EDGE data rate will be important because it will support approximately $95 \%$ of users who eventually connect to $5 \mathrm{G}$ services. Capacity is another important metric used to characterize networks. It refers to the total data rate per unit area that a network can provide. Area capacity is typically represented in bits per unit area per second. It is expected that this capacity will be increased by approximately 100 times in 5G networks. Such growth could be accomplished by incorporating various technologies into communication networks including Wireless Software Defined Networking (WSDN), massive Multi-Input Multi-Output (MIMO) systems and millimeter-wave-based communication technology (Akyildiz et al., 2016). 
Low latency: The roundtrip latency of an LTE network for an information plane is approximately $15 \mathrm{msec}$ (Fettweis, 2014). However, for recently developed applications such as tactile internet, multiplayer gaming and virtual reality which might eventually use $5 \mathrm{G}$ networks, the latency must be improved to approximately $1 \mathrm{msec}$, i.e. by at least one order of magnitude (Andrews et al., 2014). A recently introduced utility for tactile internet employs a Wi-Fi network for real-time control. In particular, the interaction time between steering and manipulation of virtual and real items is set to approximately $1 \mathrm{msec}$ to avoid cyber sickness and ensure application feasibility. Current state-of-the-art smartphones use contact or touch interfaces for interaction. However, future devices might use different interfaces including haptic, auditory and visual inputs and outputs to offer different interactions within online surroundings for programs related to healthcare services, sports, gaming and virtual reality. As users of such applications will require real-time interaction, system delays can degrade user experience (Akyildiz et al., 2016).

MTC which involves device automation is another technology that can be assisted by future $5 \mathrm{G}$ networks (Shariatmadari et al., 2015). MTC requires very low latency which is important in applications such as Vehicle-to-Vehicle (V2V) communication. The mobile wireless communication Enablers for Twenty-twenty Information Society (METIS) project has proposed the use of efficiency, traffic and safety as metrics for evaluating latency. Furthermore, in the case of intelligent traffic systems, vehicles should be able to prevent accidents via. communication in a timely manner. These and other intelligent systems are further discussed in the description of the METIS project (Osseiran et al., 2014). In addition, low latency will enhance user experience in applications such as multiplayer gaming. To reduce latency based on application requirements, technological innovation is required for appropriate waveform design. This can be achieved using WSDN which emphasizes architectural flexibility for high-layered networks (Akyildiz et al., 2016).

Low energy consumption: IoT devices should also be supported by future $5 \mathrm{G}$ networks (Al-Fuqaha et al., 2015). These devices include sensors that collect information from their surroundings and transmit it to central servers. Such devices do not require much energy and are highly cost-effective owing to their long lifespans. However, they are rarely connected to Base Stations (BSs) and are rarely turned on as their short battery lives do not always permit synchronization with BSs, a process that requires more energy than data transmission. These issues can be addressed using radio access to $5 \mathrm{G}$ networks as little to no synchronization will be required if $5 \mathrm{G}$ networks are used. In particular, the decoding power, packet-forwarding scheme and packet header length constrain IoT services (Akyildiz et al., 2016).

Furthermore, with the expected proliferation of connected smart devices, the number of BSs required will increase to support such devices adequately. Thus, the BS density will increase when small cells are deployed. Considering this expected trend, BSs must be highly energy efficient. In particular, small improvements in energy efficiency can be scaled to larger IoT networks to save energy and increase efficiency further (Akyildiz et al., 2016).

High scalability: Network scalability is an important consideration when designing new-generation wireless communication networks, given the large number of mobile devices expected to be connected to such networks. IoT devices further add to this burden. Furthermore, V2V communication whose usage is expected to increase considerably will negatively affect network scalability. It is anticipated that 50 billion devices will be connected to wireless networks by 2020 (Cisco, 2015). Scalable networks can efficiently accommodate such large numbers of devices. Furthermore, high scalability is an important factor in achieving high performance in current and future applications. Similarly, scalable cellular networks are necessary for efficient $\mathrm{V} 2 \mathrm{~V}$ communication under high traffic density (Di Taranto et al., 2014).

Network scalability requires a complete upgrade of all the necessary layers. The outer layer should include resources with sufficient bandwidth to support multiple signals and large data transfers. Furthermore, the network structure should be able to control the transmission power for channel estimation to counter interference (Akyildiz et al., 2016). The design of effective Medium Access Control (MAC) layer protocols can promote scalability and allow appropriate handling of several connected devices. Protocols developed to enhance network performance considering user environments can decrease latency and enhance spectral efficiency (Akyildiz et al., 2016). For networks and transportation on surfaces, intelligent routing algorithms are required to ensure high scalability at reliable speeds for large groups of users. Furthermore, for highly mobile users, 
vehicular networks must possess scalability and ensure user satisfaction through effective and reliable handoffs over the travel routes and through different routing algorithms based on user movement predictions. Network scalability can be achieved using WSDN and Network Function Virtualization (NFV) (Akyildiz et al., 2016; KaiTen et al., 2008).

Enhanced reliability and connectivity: "In $5 \mathrm{G}$ networks, coverage and handover effectiveness must be enhanced for improved user experience, especially, in the case of millimeter-wave spectrum usage. Considering the expected increases in the densities of BSs and linked devices as well as the introduction of picocells and femtocells, the number of handovers that a BS can handle should be increased by at least two orders of magnitude. To support this expected increase in demand, novel handover algorithms and methods are required to provide better coverage around cell edges. Another related issue involves authentication and privacy during handovers (Duan and Wang, 2015). Current approaches cause delays of hundreds of milliseconds when contacting servers for handovers which is not suitable for $5 \mathrm{G}$ applications. In addition, when too many frequency bands are used in millimeter wave spectrum-based applications, the signal transfer range is reduced. Therefore, maintaining connectivity in $5 \mathrm{G}$ systems in such cases would be difficult which could pose problems for several applications such as mission-critical services (Akyildiz et al., 2016).

Improved security: Since, 2015 wireless network security has drawn increasing attention, partly owing to the increased popularity of online payments, mobile payments and digital wallets (Akyildiz et al., 2016). Retrospective appraisal which focuses on network security has been developed to ensure wireless connectivity and protect user privacy in previous generations of communication technology. However, with the expected increase in data traffic owing to the adoption of $5 \mathrm{G}$ systems, security issues including establishing trusted connections using Authentication, Authorization and Accounting (AAA), as well as security and management of data and tasks in the cloud will need to be re-evaluated and improved. In particular, novel encryption protocols must be developed. Notably, no universally agreed-upon standard was developed for $4 \mathrm{G}$ networks to protect the private information of users. It is important for $5 \mathrm{G}$ networks to address this oversight (Akyildiz et al., 2016).
Furthermore, as 5G networks are expected to include large-scale IoT applications, AAA activities for interconnected devices should employ the best protection methods. Network controllers, device manufacturers and standardization carriers should assist one another to ensure the security of the personal information of their customers. In addition as with the internet, power grids must be used to strengthen legal regulations related to wireless network security (Akyildiz et al., 2016).

\section{MATERIALS AND METHODS}

5G technology enabling solutions: The 10 technologies described below can be considered to work together effectively in the $5 \mathrm{G}$ network architecture. Radio Access Network (RAN) architecture can be improved by implementing the following technological solutions (Akyildiz et al., 2016):

\section{. Ultra-densification \\ . WSDN \\ . NFV \\ . Device-to-Device (D2D) communication \\ . Massive MIMO \\ . Millimeter-wave and new RAN technologies}

In contrast, the primary network can be improved by using the following (Akyildiz et al., 2016):

$\begin{array}{ll}\text {. } & \text { Big data } \\ \text {. } & \text { Green communication } \\ \text {. } & \text { IoT } \\ \text {. } & \text { WSDN and mobile cloud computing }\end{array}$

WSDN: In general, incorporating flexibility into network architecture is difficult and has become a major challenge in $5 \mathrm{G}$ network design. However, Software-Defined Networking (SDN) can overcome this problem (Akyildiz et al., 2014a, b). The emergence of SDN has primarily facilitated network activities at data centers and has helped improve next-generation internet technologies (Akyildiz et al., 2014a, b; Jain et al., 2013). Its principal characteristics are separation of the data and management planes and functionalities of novel network control based on network abstraction. SoftAir (Akyildiz et al., 2015a, b) is a new WSDN architecture and equipment designed for its implementation is already available (Akyildiz et al., 2016). 
SDN and 5G network integration has been discussed in the literature from industrial and academic perspectives (Akyildiz et al., 2015a, b; Anonymous, 2016; Bansal et al., 2012; Dely et al., 2012; Anonymous, 2014a-c; Gudipati et al., 2013; Jin et al., 2013; Anonymous, 2015a-c; Suresh et al., 2012; Wu et al., 2015; Yap et al., 2010). Detailed WSDN solutions were presented by Akyildiz et al. (2015a-c) where the core factors that degrade or prevent network scalability were noted to influence all of the proposed solutions directly, except SoftAir (Akyildiz et al., 2015a-c).

First, all of the designs have incomplete architectural solutions for $5 \mathrm{G}$ structures. However, partial designs were included, e.g., software-defined RANs. SoftAir involves measurable software-defined planning which provides a crucial layout and allows access to networks using seamless OpenFlow incorporation, thus, facilitating central management of the control plane. Second, prior designs such as that of the well-known cloud-RAN (Wu et al., 2015) have limited scalability because of their coarse-grained front-haul network decoupling approach where the sinusoidal in-phase and quadrature components are amplified and modulated and are supposed to be channeled within the processing servers. Such transmission burdens the optical transport network, particularly in the case of highly advanced Wi-Fi technology. In contrast, SoftAir provides a fine-grained front-haul network structure that eliminates front-haul bottlenecks and significantly improves system capacity. Finally, in contrast to other available solutions, SoftAir includes a full control kit that enables traffic balancing, traffic classification, carrier scheduling and network virtualization, thereby, maximizing performance (Akyildiz et al., 2016).

To the best of our knowledge, SoftAir is best suited for $5 \mathrm{G}$ cellular systems because it not only improves hardware forwarding infrastructure and software algorithms but also enables flexible and efficient resource sharing. These advantages lead to high spectral efficiency, enabling different networks to converge and improving energy efficiency (Akyildiz et al., 2016).

NFV: NFV which is functionally equivalent to SDN, allows virtualization of an entire network based on a previous hardware connection and is therefore, suitable for cloud systems. The conventional network architecture involves operators purchasing and installin proprietary devices to execute network functions for every subnetwork. However, such specialized hardware is expensive and difficult to configure. Therefore, network subscribers experience low agility, leading to longer product cycles. In contrast, NFV decouples the physical hardware and its associated network functions, enabling them to be executed centrally on generic cloud servers which is advantageous in terms of flexibility and scalability. NFV decreases the capital required for the acquisition of hardware devices as well as the operational costs by aggregating resources crucial for virtual network functions that operate on a centralized server pool (Akyildiz et al., 2016).

Furthermore, NFV can be extended to IoT. IoT, together with its wide applications, involves a large number of connected devices on a single network. By using NFV, the expense associated with implementing IoT can be significantly reduced. The number of physical IoT devices on the network can be reduced by virtual implementation. For instance, in a function, virtualization is performed by a sensor (Van den Abeele et al., 2015) which enables it to distribute information among the IoT devices based on the concept of modular blocks. The details of IoT and NFV interaction will be discussed in greater detail in section 3-G. Network re-planning is time consuming and expensive in the case of inflexible mobile networks. Moreover, NFV can make vital networks more scalable, expansive, smart and flexible by virtualizing most entities (e.g., Packet data network Gateways (PGWs), mobility control entities, Serving Gateways (SGWs) and home server subscribers). In addition, it is free from the geographical disadvantages of other services. For instance, PGWs and SGWs can be co-located with $\mathrm{BSs}$, thus, eliminating the need for distantly located links. Furthermore, NFV can eliminate the bottlenecks of vital networks by merging computing resources (Akyildiz et al., 2016).

The solutions that assimilate WSDN and NFV layouts are described by Akyildiz et al. (2015a-c). Notably, owing to its complex architectural design, SoftAir (Jain et al., 2013) includes an entire solution wherein NFV is used to create a system based on a combination of NFV and WSDN. By considering NFV (network cloudification), SoftAir includes a fine-grained, front-haul network disintegration solution that reduces the amount of transferable data among Baseband Servers (BBSs) and Remote Radio Heads (RRHs) by partially processing the baseband in RRHs and directing the rest of the processing toward BBSs. Furthermore, when the Spectral Database for organic compounds (SD-BS) implements the OpenFlow interface, acquisition of systematic interfaces among distinct wireless requirements is achieved. OpenFlow allows vertical 
mobility which in turnpermits consumers touse SD-BSs alongside their distinct wireless requirements. SoftAir also, leverageshigh-performing controllers, improving network flexibility and management optimization scalability (Akyildiz et al., 2016).

Millimeter-wave transmission: As indicated by the Federal Communications Commission (FCC) and several industrial and academic research groups, millimeter-wave transmission uses large bands. Thus, it has considerable potential for realizing throughputs on the order of gigabits per second. Millimeter-wave transmission occurs in the frequency range of $30-300 \mathrm{GHz}$. However, scientists usually include the closest centimeter-wave frequency band in the $24-28 \mathrm{GHz}$ range when defining it. Numerous spectral resources are available through light-licensed and unlicensed millimeter-wave frequency bands which support systems that provide high data rates, low latency and flexible connectivity to many users (Akyildiz et al., 2016).

In October 2015, the FCC proposed some rules for the release of millimeter-wave spectral bands for $5 \mathrm{G}$ services (Anonymous, 2015a-c). The frequency bands authorized for mini-mobile platforms are the $39 \mathrm{GHz}$ band (from $38.6-40 \mathrm{GHz}$ ) and $28 \mathrm{GHz}$ band (from 27.5-28.35 GHz). A $60-\mathrm{GHz}$-based Wireless Local Area Network (WLAN) that was allocated for IEEE 802.11 ad has shown great potential for supplying high data rates in short-scope (especially, indoor) environments; Hence, the FCC proposed the use of the 64-71 $\mathrm{GHz}$ band for such short-radius activities. This $71 \mathrm{GHz}$ band not only increases the bandwidth for WLANs but also improves WLAN service data rates. In addition, the FCC proposed a hybrid-licensing scheme which allows carriers that provide services in the $37 \mathrm{GHz}$ band to perform decision-making. The hybrid band ensures broadband network improvement and can supplement traditional mobile networks (Anonymous, 2014).

The current requirements of 4G LTE channel models include many frequently learned propagation environments such as indoor shopping malls/centers, indoor offices, highways, highly populated metropolitan environments and rural places. The infrastructure arrangement of wireless communication networks is based on the environment in which the system is located. The arrangement includes the configurations of the primary channel specifications, the definite mechanical and electrical down-tilt angles of the BS sector antenna and a number of systematic aligned arrays that should be in the antennas of all sectors for both the BS height and the estimated inclusion of sectors. In future, wireless systems, novel mobile arrangements could be used to fulfil customer requirements. Modifications to propagation-based infrastructures should consider direct communication among devices that involve multiuser communication scenarios, millimeter waves and autonomous vehicular communication using millimeter waves (Akyildiz et al., 2016).

Massive MIMO: In addition to millimeter-wave communication, massive $\mathrm{MMMO}$ is a key technology that could be incorporated into $5 \mathrm{G}$ network solutions. In contrast to multiuser MIMO used in $4 \mathrm{G}$ network structures where tens of aerial components are installed at the BSs and consumer terminals, the massive MMMO layout includes several groups of antennas around the BSs to account for the growing volume and required system throughput, thus, allowing greater freedom in terms of scalability (Larsson et al., 2014). In massive MIMO, TDD and spatial multiplexing are employed for multiple-user spaces with accessible time-frequency assets. The three viable massive MIMO designs for BS antenna arrays are cylindrical, rectangular and linear designs. Notably, the use of rectangular and linear arrays is more viable on high-rise building surfaces for indoor customers at different heights or for outdoor pedestrians. In addition, the rectangular design allows access to many customers via. spatial multiplexing. In contrast, cylindrical arrays are used for moving consumers and are deployed to monitor their travel routes (Akyildiz et al., 2016).

Massive MIMO has several benefits, including increased spectrum efficiency, reduced latency and scalable structured air interfaces. Owing to the presence of more antenna elements at the BSs than at the mobile terminals, the pre-coding steps are simple, leading to a considerable increase in spectrum utilization efficiency (Marzetta, 2010; Rusek et al., 2013). Furthermore, latency reduction is realized because of the numerous antennas in massive $\mathrm{MIMO}$ architecture, countering the channel dependency on frequency and ensuring that frequency-selective fading does not affect signal strength. Therefore, no stream of transmitted data experiences latency reduction due to channel distortion. In addition, owing to the numerous components of the antennas, the design of the primary air interface structure is flexible and/or scalable to the traffic conditions of real-time data. For instance, if a mobile device accessing a massive-MIMO BS has several other consumer terminal connections not all of the elements in the BS antenna need to be active simultaneously. Therefore, the energy consumption of each element can be reduced to the least possible value while still satisfying the operational requirements. In contrast, in the case of 
heavy data traffic in the range of a single massive-MIMO mobile device all of the components can become active to ensure rapid data transmission to the consumers. Researchers who have studied $5 \mathrm{G}$ architecture are very interested in the scalability benefits provided by massive $\mathrm{MIMO}$ and believe that it will play an important role in the development of $5 \mathrm{G}$ networks (Akyildiz et al., 2016).

Ultra-densification: The traditional macro-mobile network architecture is the foundation of $4 \mathrm{G}$ networks. It was estimated that the worldwide monthly mobile data traffic would exceed 15 exabytes by 2018 (Osseiran et al., 2014) due to the increased use of smartphones, tablets wearable devices and other mobile devices which require adequate bandwidths for streaming videos and roaming data. Considering unforeseen developments in cellular device types as well as services and applications, 5G cellular networks should be highly flexible in every network layer. However, high-tower-mounted traditional BSs cannot fulfil such demand increases. Thus, the two most highly recommended propositions for accommodating the ever-increasing number of consumer terminals and unloading some data flows which are already congested in the $4 \mathrm{G}$ BSs are spectral aggregation and spatial densification. These approaches can facilitate the development of additional mobile layers to use spectral resources that can be efficiently shared, thereby increasing capacity. In particular, spatial densification depends on Heterogeneous Network (HetNet) principles which were first introduced in the 4G LTE system. Smaller element groups for HetNets including femtocells, picocells and well-dispensed antenna elements can be of great importance. Furthermore, spectral aggregation counters can address the challenges of non-continuous bandwidth fragmented resources and shared use of the spectrum to control licensed and unlicensed spectra, thus, providing higher efficiency (Akyildiz et al., 2016).

Big data and cell cloud computing: Data storage techniques also need to be modified for $5 \mathrm{G}$ networks to allow higher network throughput. Traditional data storage on local devices is insufficient to handle substantial increases in data caching requirements, considering user requirements for large file downloads and $\mathrm{HD}$ video streaming. Recently, cloud storage has drawn considerable attention because it enables convenient data recovery. Consumers can upload data to the cloud via. the internet, thus, saving storage space on local devices (Akyildiz et al., 2016).

In cellular devices and networks, the amount of generated data highlights the significance of big data analysis. Currently, data analyses are executed on different physical machines using platforms such as Apache Hadoop (in-house Hadoop). This platform requires infrastructure setup and regular manual maintenance. Moreover, reconfiguration is often expensive and results in reduced efficiency. However, efficiency, cost and scalability benefits are greater in large data cloud computing than in in-house Hadoop. Big data analysis is expected to develop into an efficient and powerful approach owing to benefits in terms of software-defined switching and control based on WSDN (Akyildiz et al., 2016).

Big data and cellular cloud computing offer flexibility to $5 \mathrm{G}$ networks along with highly efficient data control. Cellular cloud computing will increase the speed of data control and file sharing over cloud servers. In contrast, to cellular systems in which the downlink quality is important for system performance, cellular cloud computing depends on the uplink quality. Specifically, back-haul cellular cloud computing depends on the uplink quality while the back-haul itself depends on the cloud server. Consequently, traffic-smart data routes need to be provided for back-haul gateways (Kaewpuang et al., 2013).

IoT: IoT network devices include physical objects, buildings, vehicles and everyday appliances. Examples of such devices include microwave ovens, health monitors and smart watches. Such devices send sensed information to remote databases through the internet. A server can also control the appliances. The collected information is processed to understand the underlying processes in the devices. Such information is useful for building smarter systems. This approach can be adopted to develop smart environments including smart homes, smart cities and towns, smart transportation systems and smart healthcare systems (Vermesan and Friess, 2013). Owing to its potential applications in various areas, IoT could promote internet connectivity in many devices. This shift from consumer-connected concepts to connections to other entities will result in a significant improvement in the number of devices in these network systems. Owing to the sheer volume of expected IoT devices as well as their various applications, IoT will be of great importance in designing $5 \mathrm{G}$ systems (Akyildiz et al., 2016).

D2D communication: In conventional cellular networks, most communication occurs between BSs and cellular devices. Even when two users in a range are performing explicit communication, a link is created 
via. a BS. However, this process is inefficient for real-time services that require high data rates and low latency. The concept of improving spectrum efficiency using direct communication between devices was proposed by Lin and Hsu (2000). It involves developing multihop relays alongside cellular network devices. Since then, many D2D communication systems have been researched and have become parts of LTE-A networks. These systems have been proposed for licensed and unlicensed spectral bands. Further, detailed information regarding the classification and characteristics of D2D communication was presented by Asadi et al. (2014). It is expected that $5 \mathrm{G}$ networks will continue offering support for applications requiring explicit communication connections (Tehrani et al., 2014). Such applications include games, social networks and file sharing (Bangerter et al., 2014). Furthermore, D2D communication will enhance the QoS of mobile networks because relaying can effectively increase data transfer rates for consumers, thereby ensuring traffic alleviation at the BSs and nearby devices which could unload the BSs and subsequently improve customer experiences. In summary, D2D communication provides three advantages: proximity gain due to the shortened distance between the transmitter and receiver, allowing high data transfer rates with lower power consumption gain reuse which refers to spectral resource reuse at different locations when the communication range is short and hop gain through a single connection in D2D communication based on uplink and downlink requirements in BS-based regular communication (Fodor et al., 2012).

D2D communication can provide a platform for many inventive applications and services. For example, a crucial application involves public safety and security (Fodor et al., 2014). In mountainous areas and long tunnels which are susceptible to landslides as well as in zones that experience earthquakes, mobile networks could fail or be unavailable. In such cases, D2D communication can be useful for relief and rescue missions. Furthermore, D2D communication can be applied to MTC, particularly in $\mathrm{V} 2 \mathrm{~V}$ communication where it can be used to retrieve accident information and warn self-driving cars. For example, D2D communication was included in 3GPP Release 12 for proximity services to enhance public safety (Lin et al., 2014). Furthermore, D2D communication is useful for connecting devices such as smart watches to smartphones. Although, this type of connection can be achieved via. Bluetooth, a unified protocol will lead to reduced costs and power consumption considering the device design and usage. Furthermore, D2D communication is more reliable because Bluetooth is not intended for high-mobility environments. D2D communication could also lead to the development of advanced social networking applications (Akyildiz et al., 2016).

Green communication: Over the next decade, the number of devices linked to wireless networks and the volume of data generated by these devices are likely to increase 100 and more than 1000 times, respectively. Such growth will pose major challenges for which affordable and sustainable approaches should be considered. Even though mobile communication currently accounts for less $1 \%$ of the global carbon footprint, it should be reduced further. Operators have already been forced to consider power charges as important components of their operational expenses. Therefore, reduced energy consumption and a shift to green communication are important from environmental and economic perspectives (Akyildiz et al., 2016).

Radio access techniques: Section 2 stated that the current underlying techniques for radio access need to be redesigned to support the expected higher data rates of $5 \mathrm{G}$ networks. Furthermore, because of spectrum scarcity and associated costs, improvements in spectral efficiency should be considered. Many applications that will partially utilize $5 \mathrm{G}$ networks such as tactile internet, require latency as low as $1 \mathrm{msec}$. This requirement constrains radio access technique design. In addition, reduced latency should be realized when the number of layers is greater. Similarly, IoT applications include contexts in which device connection to BSs is time-limited. The power constraints of such devices require them to research with fully synchronized BSs. The employed multiple-access technique supports systems with no or loose BS synchronization.

As with any other wireless system when the multiple-access technique is employed for radio access, interference becomes a problem. Thus, the selected approach should have minimal Out-of-Band (OOB) emissions to minimize the guard bands among those of the other carriers. Reduced interference requires a lower guard bandwidth which effectively increases the spectral efficiency. In addition, the numerous access methods should efficiently utilize the multiple existing antennas present in most devices. Thus, when a multiple-access method is selected, its compatibility with MIMO technology must be considered. Finally, the selected 
radio access technique must be energy efficient (Akyildiz et al., 2016). Radio access technologies can be mainly classified as being based on full-duplex communication approaches and multiple-access methods (Akyildiz et al., 2016).

Full-duplex communication: Full-duplex communication systems can simultaneously transmit and receive signals in the same frequency band. This process represents a major shift from previous half-duplex communication systems in which FDD or TDD was used to realize duplex communication. The 4G LTE RAN technology evolved into the Evolved-Universal Terrestrial Radio Access Network (E-UTRA) which provides support for FDD and TDD operation modes (Anonymous, 2016). However, both modes suffer from low efficiency and performance in the cases of obsolete Channel State Information (CSI), guard intervals and band requirements for frequency and inflexible bandwidth allotment (Mahmood et al., 2015). Even though full-duplex systems overcome these disadvantages these modes were found to be infeasible for the implementation of a practical full-duplex system because such a system would suffer from considerable self-interference from the transmitter which would obscure the signals to be received. Current full-duplex systems have been realized by cancelling such self-interference (Akyildiz et al., 2016).

The proposed self-interference cancellation techniques involve interference suppression at different stages of the receiving chain (Zhang et al., 2015). For example, the propagation-domain technique of suppressing interference involves protecting amplifier input of radio frequency from overwhelming self-interference. In contrast, the cancellation of analogue-domain interference ensures that Analogue-to Digital (A/D) converters are not overpowered by self interference whereas the cancellation of digital-domain interference eliminates residual self-interference resulting from nonlinearities in the $\mathrm{A} / \mathrm{D}$ converters and noise in the oscillator phase. Some articles have reported on the combinations of these methods to attain superior self-interference cancellation (Akyildiz et al., 2016).

Full-duplex communication can be utilized by $5 \mathrm{G}$ cellular networks to increase their capacities in two ways (Zhang et al., 2015). First, when the User Equipment (UE) transmits data to a BS or another device using D2D communication, the BS or other device can simultaneously transmit its own data to the same UE. The BS and UE can decode the collected data while simultaneously transmitting them in the same frequency band. This operating mode is known as bidirectional transmission. Second, unidirectional transmission similar to full-duplex relaying can be implemented (Alves et al., 2015). In this method, data are transmitted from the first to the second and then to the third node. Although, one might expect the spectral efficiency in full-duplex communication to be twice the attainable spectral efficiency, the increase in throughput is approximately $30-40 \%$ in practice (Mahmood et al., 2015). Thus, these methods can be improved further.

Although, self-interference cancellation is the primary problem to be addressed when implementing full-duplex communication it is not the only issue. The entire current stack network has been developed using half-duplex systems and this characteristic must also be considered. Therefore, network performance needs to be reassessed and redesigned to attain true full-duplex communication. For instance, a water-filling algorithm would be useful for power allocation to boost the spectral efficiency of a half-duplex system. However, because it does not account for self-interference, it is not optimal for a full-duplex system. Furthermore, some MAC conduct rules applied in previous systems such as carrier access systems, innately assume the systems to be half-duplex. Therefore, they cannot be used directly for full-duplex systems. Thus, as proposed by Zhang et al. (2015), the development of novel MAC protocols for full-duplex systems is important. In addition, redesigning the application layers of full-duplex systems is crucial (Akyildiz et al., 2016).

Multiple-access techniques: For the downlink channel, E-UTRA uses orthogonal FDMA (OFDM) whereas for the uplink channel, Single Carrier-FDMA (SC-FDMA) is employed to conserve power and reduce the Peak-to Average Power Ratio (PAPR) (Anonymous, 2016). Although, the techniques employed in 4G LTE networks work smoothly they will suffer setbacks under the numerous new conditions in 5G networks. This problem should be considered when designing $5 \mathrm{G}$ networks. The different multiple-access techniques that can be considered for $5 \mathrm{G}$ structures presently are discussed.

OFDM: Various advantages such as structural simplicity, robustness against frequency-selective fading channels, ease of implementation, compatibility with $\mathrm{MIMO}$ and efficient execution using Fast Fourier Transforms (FFTs) have promoted the widespread application of the OFDM 
technique in numerous frameworks involving broadband over power lines and IEEE 802.11 wireless LAN. Moreover, OFDM is the best-known multicarrier modulation and multiple-access technique for 4G LTE (Akyildiz et al., 2016).

However, OFDM suffers from several disadvantages. It requires perfect synchronization because of the orthogonality among subcarriers which is difficult to attain in uplink and downlink frameworks (Morelli et al., 2007) because the BSs cooperate to dispatch information (Banelli et al., 2014). Furthermore, low latency cannot be achieved using OFDM because of the requirement of a Cyclic Prefix (CP). In addition, OFDM suffers from a high $\mathrm{PAPR}$, pilot contamination and high $\mathrm{OOB}$ emission. Several studies have been conducted with the objective of overcoming these drawbacks (Farhang-Boroujeny, 2011). For example, Schaich et al. (2014) proposed a method of minimizing the side lobes and thus, reducing pilot contamination was proposed. However, no study has been able to address all of the above-mentioned issues.

Filter Bank Multicarrier (FBMC) technology: The FBMC approach can be considered a generalized version of OFDM. It involves transmitting sets of data through modulated filter banks. FBMC systems individually filter each subcarrier whereas OFDM involves filtering the complete band. FBMC systems replace the sync-pulses of subcarriers in OFDM systems with different shapes. A filter design that satisfies the required spectral characteristics can be developed via. freedom in filter shape selection. FBMC devices are classified as staggered and filtered multitone carriers between which the former are more popular (Schaich and Wild, 2014).

By using different prototype filters, FBMC technology can achieve good frequency localization. In particular, the spectral efficiency of a wireless system increases because $\mathrm{CP}$ is eliminated. Polyphase filter structures, Inverse FFT (IFFT) and Feature Triangle Tracking (FTT) blocks can be used to implement FBMC systems efficiently. Furthermore, symbol synchronization is not required because FBMC is useful in cases of frequency offset (Akyildiz et al., 2016).

Nevertheless, FBMC systems have some disadvantages. First, they cannot use other forms of modulation and only research with offset Quadrature Amplitude Modulation (QAM). As with OFDM, FBMC technologies suffer from high PAPRs. Although, high spectral efficiency can be provided by an FBMC system when there are numerous transmitted symbols, the spectral efficiency decreases at the ramp-up and ramp-down times at the ends of the intervals when filtering is performed and the transmission time is on the order of milliseconds. This issue is important in applications such as IoT and MTC because the packet sizes in those applications are small. Furthermore, pulse-amplitude modulation is the only type of modulation supported by some FBMC systems. Higher-order modulation techniques such as 64-QAM cannot be used because of this restriction. In particular, 64-QAM is employed in the LTE E-UTRA approach and provides limited data-rate support. In addition, FBMC systems are more error-sensitive in terms of channel measurements, especially when errors are present in CSI FBMC modulation which results in lower efficiency compared with the modulation provided by OFDM. Inter-Symbol Interference (ISI) and Inter-Carrier Interference (ICI) are caused by these imperfections. Consequently, FBMC is less compatible with MIMO systems than OFDM systems. Moreover, FBMC technology cannot be used with Alamouti space-time block codes and spatial multiplexing with maximum likelihood detection which are advanced wireless methods for information transmission (Akyildiz et al., 2016).

Universal Filtered Multicarrier (UFMC) technology: UFMC technology is a combination of the OFDM and FBMC technologies. As mentioned previously, the difference between the OFDM and FBMC techniques is that the entire band is filtered in the former whereas each subcarrier is individually filtered in the latter. In contrast, groups of subcarriers are filtered in UFMC systems to minimize OOB emissions. UFMC systems split data streams into several subsystems. Then, a pulse-shaping filter is applied to each subsystem. Therefore, the UFMC technique is the most successful in reducing interference from adjacent subbands. Furthermore, the subsymbols are shortened because of the frequency subband aggregation which increases the bandwidth. Consequently, UFMC systems are more suitable than OFDM and FBMC systems for applications requiring short-packet transmission. UFMC systems that do not need CPs also increase the spectral efficiency. Moreover, inherent protection against ISI is provided by the filter ramp-up and ramp-down periods at the beginnings and ends of the frames, respectively. In the presence of Carrier Frequency Offset (CFO) and under both imperfect and perfect CSI conditions, UFMC systems outperform OFDM systems. Thus, UFMC is useful for coordinated multipoint 
transmission where achieving synchronization among transmitters is difficult. Furthermore, MIMO systems are supported by the UFMC technique (Akyildiz et al., 2016). Despite these advantages, the UFMC equalization method is complex and cannot be implemented in devices that are highly power constrained or in those with limited computational abilities. In addition, UFMC systems are sensitive to small timing misalignments, rendering them unsuitable for deployment in MTC and IoT applications which require loose or no synchronization. Therefore, even though UFMC technology has several advantages over OFDM and FBMC technologies, it does not meet all of the requirements for use in $5 \mathrm{G}$ mobile networks (Akyildiz et al., 2016).

Generalized FDM (GFDM): GFDM was first proposed for applications involving a high degree of spectrum fragmentation, e.g., spectral holes that are present in television bands. Its advantageous properties have rendered it one of the best waveform options for $5 \mathrm{G}$ mobile networks (Michailow et al., 2014). GFDM is a block-based multiplexing approach in which each independently modulated block is composed of many subsymbols and subcarriers. Time and frequency domain-shifted branches of the existing and prototype filters are used to filter these subcarriers (Akyildiz et al. 2016). The GFDM approach can be considered a single-carrier frequency-domain equalization method as well as a generalization of the OFDM approach. In this approach, only one symbol is transmitted per carrier in a block in contrast in the OFDM approach, a block consists of several subcarriers.

Furthermore, only one subcarrier in the entire frequency band is involved in the single-carrier frequency-domain equalization and serial transmission of several symbols. To meet the demands of different applications, the number of subsymbols in each subcarrier, number of subcarriers and prototype filter structure can be modified. In GFDM, a CP can be added only once in a block. GFDM provides higher spectral quality than OFDM (Michailow et al., 2014) because each block has several subsymbols that are serially transmitted. For applications that involve loose or no synchronization, the need for only one CP per block can be exploited by using a sufficiently long CP. Furthermore, the use of a CP can be entirely avoided by including a window function together with smooth ramp-up and ramp-down at the edges of the time block. Most studies related to timing estimation and synchronization are based on OFDM systems because GFDM waveforms correspond to OFDM waveforms (Akyildiz et al., 2016).
The primary drawback of GFDM systems is their computational complexity. Furthermore, unlike OFDM and FBMC systems, GFDM systems cannot employ FTT/IFFT blocks for modulation and demodulation. In addition, OFDM systems are less sensitive to $\mathrm{CFO}$ than GFDM systems. GFDM systems may have limited applications as they cannot be used in several IoT devices because of their complexity. Owing to their inherent non-orthogonality, GFDM waveforms have higher bit error rates than OFDM waveforms. Moreover, although, interference cancellation techniques have been proposed, the deployment of smooth cancellation techniques would only increase the computational complexity (Akyildiz et al., 2016).

Review of GFDM and OFDM Device Models: The OFDM technique is highly sensitive to ICI. In addition, the CP is not efficient within the spectrum and stalls, if it is considerably shorter than the delay distribution inside the channel, leading to ISI. OFDM is a well-studied technique that is widely used in many wireless and state-of-the-art data-transmission methods. In the OFDM approach, a sum of subcarriers with various stages is used, resulting in a high PAPR and high OOB emission because a rectangular pulse-shaping filter is used at the transmitter (Ramadhan, 2015).

An important technological advancement in OFDM is passband leakage reduction. The OFDM approach requires only a single modification to the final structure while in the GFDM approach, frequency and time-based pulse-shaping optimization are adopted for spectral and energy efficiency. Consequently, more than one symbol can be transmitted which is consistent with a multicarrier system in a two-dimensional block structure (frequency and time) using GFDM.

Each subcarrier is circularly convolved using a shaping pulse to obtain the block structure. The transmitted signals are appropriately placed in the subcarrier frequency range which is the most successful for the radiation inside the stopband because of the pulse-shaping filters. In addition, small-CP time-windowing systems may be implemented in the GFDM blocks to control leakage inside the stopband more effectively, leading to ICI and ISI production.

As GFDM systems permit the use of layouts with simple transmitters, the need for synchronization is eliminated, thus, lowering the signaling load. These GFDM properties, together with the reduced processing at BSs should contribute to reduced power 


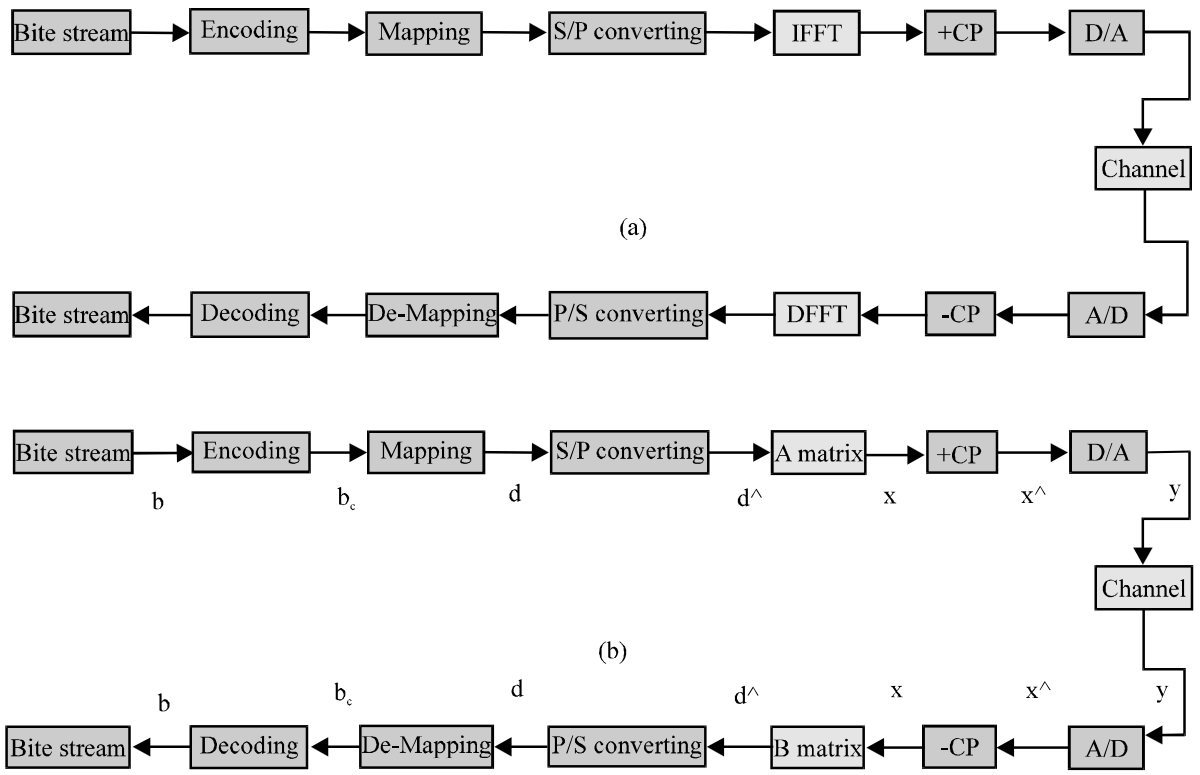

Fig. 1: General block diagrams of OFDM and GFDM transceivers: a) OFDM and b) GFDM

expenditure at the terminals. However, the signal design in the GFDM approach leads to self-interference which is similar to the issue suffered by $4 \mathrm{G}$ networks in certain access scenarios. Furthermore, time-windowing systems cannot be implemented using GFDM because of sync-pulse leakage and GFDM systems needs to be synchronized such that the orthogonality among the subcarriers is maintained. As a generalized version of OFDM, GFDM is the most effective in cases in which a single subservice image is required.

The notation $\mathrm{KM}$ is used to represent samples contained in a GFDM system where $\mathrm{K}$ refers to the carriers and $\mathrm{M}$ refers to the symbols that are appropriate for minimum latency and minimum time synchronization. As specified previously, a GFDM system involves only a single $\mathrm{CP}$ following the $\mathrm{KM}$ symbol block, thus, facilitating spectral efficiency improvement and $\mathrm{MIMO}$ configuration which are more important $5 \mathrm{G}$ community parameters than frequency localization, simplicity and robustness against CSI errors. The block diagrams of OFDM and GFDM systems are shown in Fig. 1. The principal differences between these two systems are in the IFFT and Discrete FFT (DFFT) stages. In the GFDM system, the A matrix replaces the IFFT degree and the $\mathrm{B}$ matrix (Zero-Forcing (ZF) equalizer) replaces the DFFT stage.

OFDM systems: In the OFDM approach, multiple carriers are employed without using complex equalization filters which are applied in SC-FDMA structures. In addition, a guard interval system is used to reduce the bandwidth consumption and avoid ISI while ensuring that the data rate is higher than that in an SC-FDMA structure. The general block diagram of an OFDM system is provided in Fig. 1a. The data bit stream that is sent by the sender is passed to the encoding stage (in which a high rate is split into lower rates), mapping stage (in which the digital data are converted into complex form), S/P conversion stage (in which serial-to-parallel conversion is performed), IFFT stage (in which the subcarriers are made orthogonal) and CP stage (in which a copy of the end part of the symbol is made and inserted at the head of the sequence as a guard between the symbols to reduce the multipath effects and ISI). Thus, the symbol length is increased (it must be greater than channel delay spread) and consequently, the time delay and time synchronization increase. Next, the data are passed to the D/A stage (in which the digital data are converted into a continuous analogue signal) and then sent to the destination (receiver) through the channel. At the receiver side, the data are passed through the reverse processes performed at the transmitter side.

GFDM systems: GFDM is an example of a flexible, multicarrier system. Figure 1 shows that the difference between OFDM and GFDM is that the IFFT and FFT stages are replaced with the A and B matrix stages, respectively. In addition, Fig. $1 \mathrm{~b}$ clearly shows that similar 


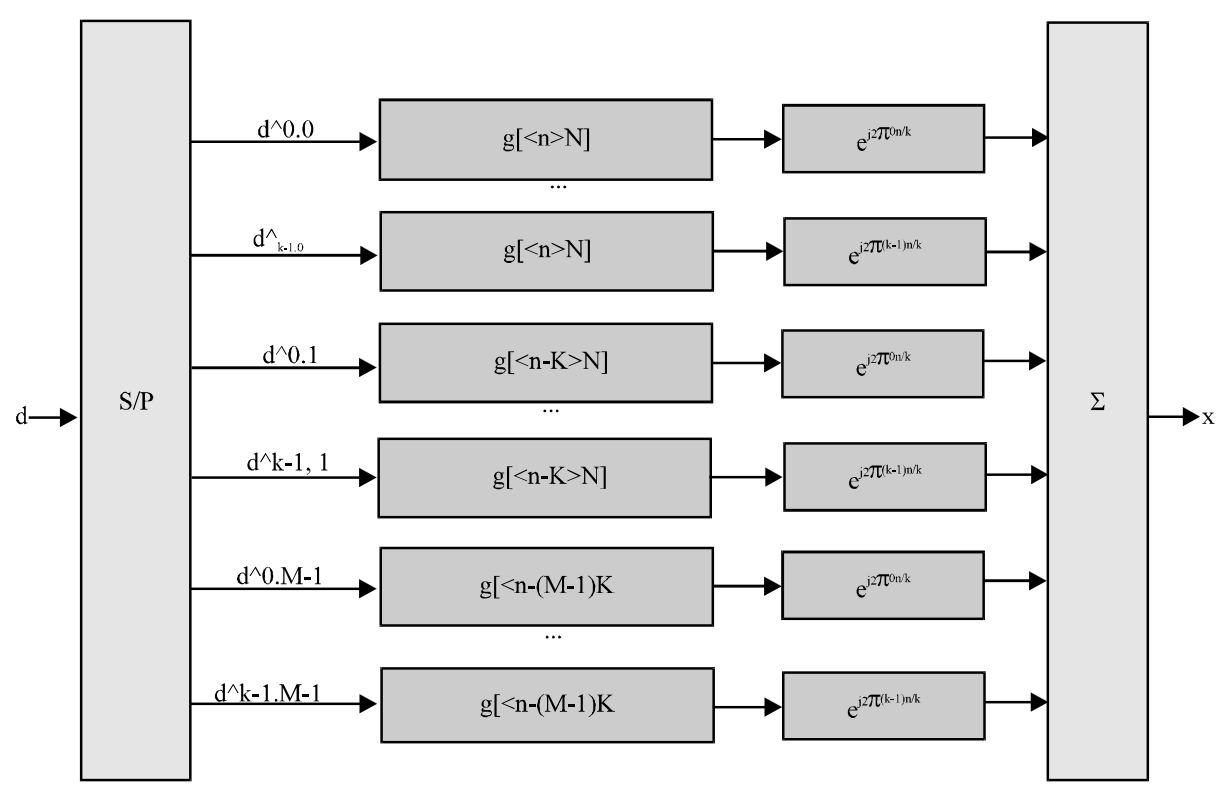

Fig. 2: Modulator-matrix block diagram

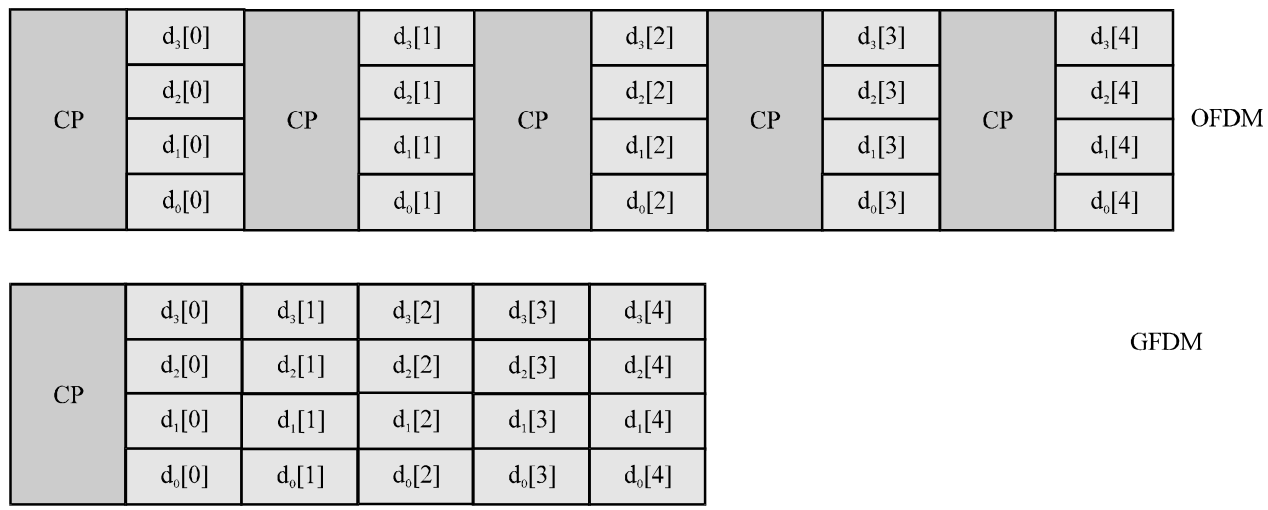

Fig. 3: General frame structures of the OFDM and GFDM systems

operations are performed in GFDM and OFDM in the first three modulation stages. After encoding (b.), mapping (d: spreads complex data symbols $\mathrm{d}(\mathrm{k}, \mathrm{m})$ through $\mathrm{K}$ carriers and $\mathrm{M}$ symbols) and $\mathrm{S} / \mathrm{P}$ conversion $\left(\mathrm{d}^{\wedge}\right)$, the data $\left(\mathrm{d}^{\wedge}\right)$ are passed to the $A$ matrix stage (in which each subcarrier is shaped with a transmit pulse filter $\mathrm{g}(\mathrm{n}-\mathrm{mN})$ and modulated with the center frequency of subcarrier $\mathrm{e}^{-\infty}$ ) which makes the data a block of carriers and symbols $(\mathrm{K} \times \mathrm{M})$ as shown in Fig. 2-4:

$$
\mathrm{d}^{\wedge}=\left[\begin{array}{ccc}
\mathrm{d}^{\wedge}(0,0) & \cdots & \mathrm{d}^{\wedge}(0, \mathrm{M}-1) \\
\vdots & \ddots & \vdots \\
\mathrm{d}^{\wedge}(\mathrm{K}-1) & \cdots & \mathrm{d}^{\wedge}(\mathrm{K}-1, \mathrm{M}-1)
\end{array}\right]
$$

Here, $d^{\wedge}$ is a complex symbol of information. Matrix $\mathrm{K} \times \mathrm{M}$ will be addressed as an information block. Hence, $\mathrm{k}=0, \ldots, \mathrm{K}-1$ represents the subcarrier and $\mathrm{m}=0$, ..., M-1 represents the time slot. To apply the Nyquist criteria, every symbol is sampled $\mathrm{N}$ times which gives $\mathrm{MN}$ samples per subcarrier. Filter $\operatorname{gTx}(\mathrm{n})$ is chosen to have $\mathrm{MN}$ periodicity. The A matrix ( $(\mathrm{n}-\mathrm{mN})$. $\mathrm{e}^{\left.\mathrm{m}-)^{-}\right)}$is multiplied times the modulated input signal $\left(\mathrm{d}^{\wedge}\right)$ to achieve GFDM modulation. After, the data symbols in the $\mathrm{d}^{\wedge}(\mathrm{k}, \mathrm{m})$ column are located and followed by upsampling, circular convolution with superposition calculations, up-conversion and pulse shaping, the following equation is obtained:

$$
\mathrm{A} \times \mathrm{d}^{\wedge}=\mathrm{x}
$$



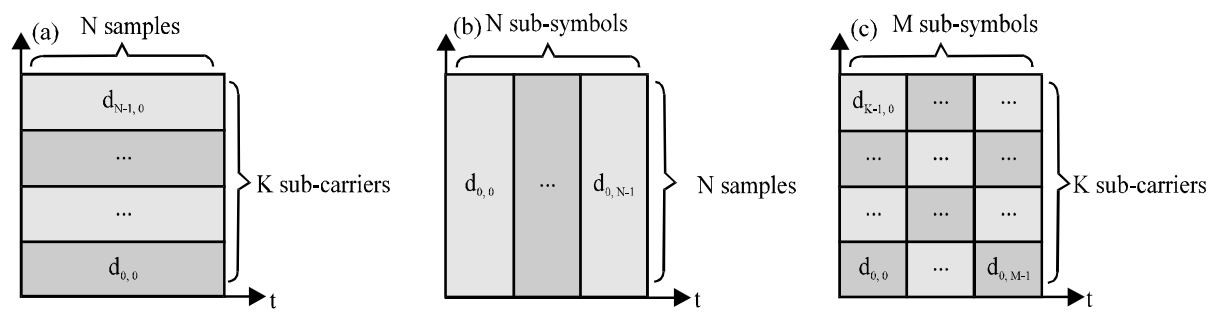

Fig. 4: Frequency and time partitioning in the OFDM, SC-FDMA and GFDM systems: a)OFDM; b) SC-FDM and c) GFDM

where, $\mathrm{x}$ contains the samples transmitted in time $\mathrm{x}(\mathrm{n})$ and the transmitted signal is as follows:

$$
x(n)=\int_{m=0}^{M-1} \int_{k=0}^{K-1} d^{\wedge}(k, m) \cdot g(n-m N) \cdot e^{j 2 \pi \frac{k n}{N}}, 0 \leq n \leq N M
$$

To equalize the frequency domain in the receiver before broadcasting, $\mathrm{CP}\left(\mathrm{N}_{\circ}\right.$ length $)$ is added to obtain:

$$
[x(N-N C P: N-1) T, x T] T=x^{\wedge}
$$

Both GFDM and OFDM have the same CP length but in OFDM, the CP is added between every two symbols to reduce the ISI whereas in GFDM, it is added between every two frames to reduce the Inter-Frame Interference (IFI) as shown in Fig. 3. Let, y represent the modulated data (after exchange with a continuous analogue signal) that are transmitted through the radio channel to the destination. Then, $\mathrm{y}^{\wedge}$ represents the received signal and can be represented as follows:

$$
\mathrm{n}(\mathrm{n})+\mathrm{x}^{\wedge^{*}} \mathrm{~h}(\mathrm{n})=\mathrm{y}^{\wedge}
$$

where, ${ }^{*}$ represents the convolution in discrete time, $\mathrm{n}$ is a vector of the Gaussian noise samples with an average of zero and variance of :., $h(n)$ represents the impulse response and $\mathrm{x}^{\wedge}$ represents the broadcasted signal with $C P$. If $h(n)$ is known at the receiver and the effects of noise interference $n(n)$ are ignored, the equation after $\mathrm{A} / \mathrm{D}$ conversion is:

$$
\mathrm{y}^{\wedge}=\mathrm{x}^{\wedge}
$$

Then, after removing the $\mathrm{CP}$, we obtain:

$$
\mathrm{x}=\mathrm{x}^{\wedge}\left(\mathrm{N}_{\mathrm{CP}}: \mathrm{N}_{\mathrm{CP}}+\mathrm{N}-1\right)
$$

When weighting is performed using the ZF matrix ( $\mathrm{B}_{\text {w }}$ $=\mathrm{A} \cdot$ ), the following signal can be derived:

$$
\left(B_{Z F}\right) \cdot(x)=\left(A^{*} d^{\wedge} \cdot(A-1)=d^{\wedge}\right.
$$

The above-mentioned steps can be performed in reverse at the receiver to obtain the transmitted bit stream (b). According to, the discussion presented above regarding the transmitter and receiver, it can be concluded that the difference between the GFDM approaches and the OFDM or SC-FDMA structures is that the former allow given time-frequency resources to be divided into $\mathrm{K}$ subcarriers and $\mathrm{M}$ subsymbols as shown in Fig. 4.

Consequently, it is possible to select the spectrum based on the platform requirements and perform pulse shaping accordingly for every subcarrier. By using either a large number of slim band subcarriers for OFDM systems or a small number of subcarriers with large individual bandwidths for the SC-FDMA structures, the GFDM approach can be used to cover part of the band width without changing the sampling rate. Further, although, filters are used, the GFDM approach is primarily a block-based technique. This property is important for individual scheduling in many different situations to achieve low-latency transmission.

\section{RESULTS AND DISCUSSION}

We used MATLAB to simulate GFDM signals. The parameter values are listed in Table 2. The Symbol Errors Rates (SERs) obtained for the OFDM and GFDM signals when the Signal-to-Noise Ratio (SNR) was $15 \mathrm{~dB}$ and 20 $\mathrm{dB}$ are presented in Fig. 5a, b, respectively. The OFDM spectra, together with the GFDM spectra were obtained in our simulations for 512 and 1024 subcarriers and are presented in Fig. 6a, b, respectively. The results demonstrate that the GFDM approach reduces the $\mathrm{OOB}$ emission to a greater degree than the OFDM approach. 

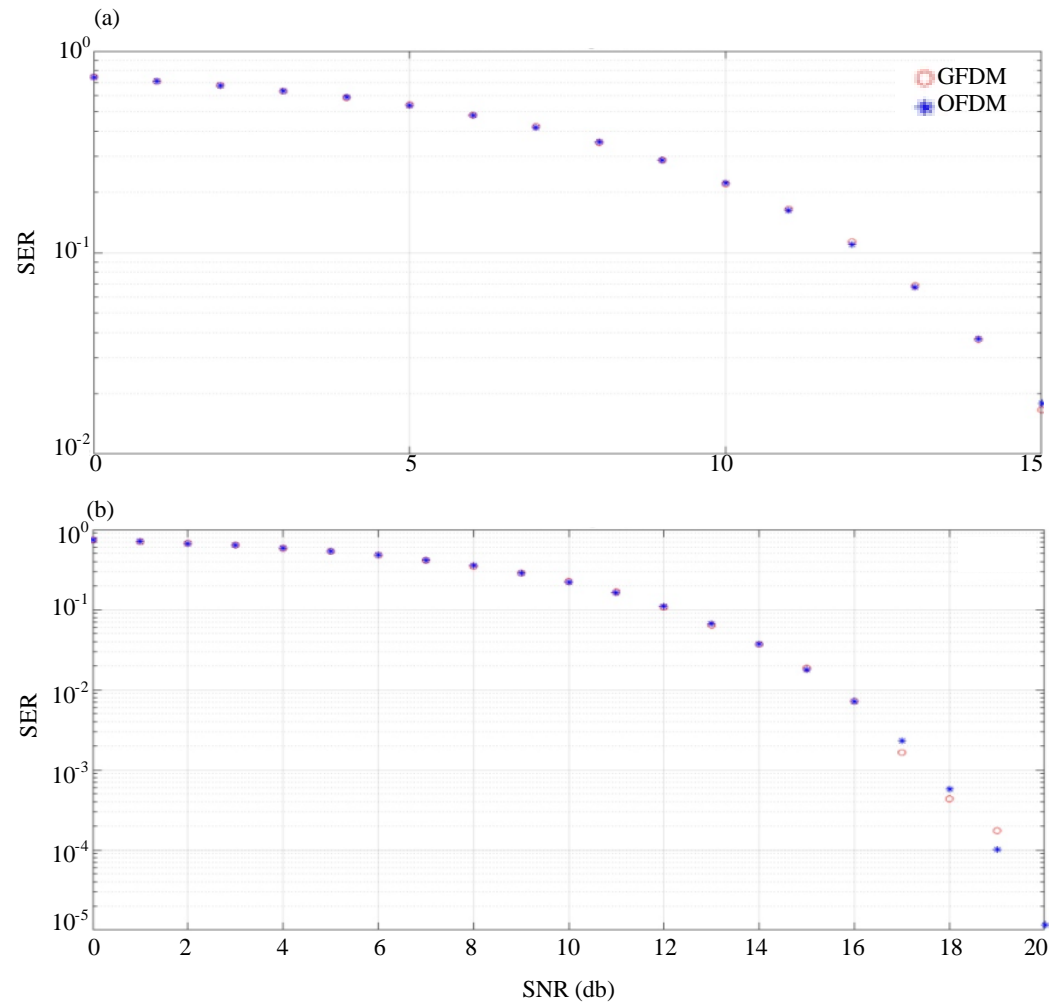

Fig. 5: SERs of the OFDM and GFDM signals: a) SERs obtained with $\mathrm{SNR}=15 \mathrm{~dB}$ and b) SERs obtained with $\mathrm{SNR}=$ $20 \mathrm{~dB}$
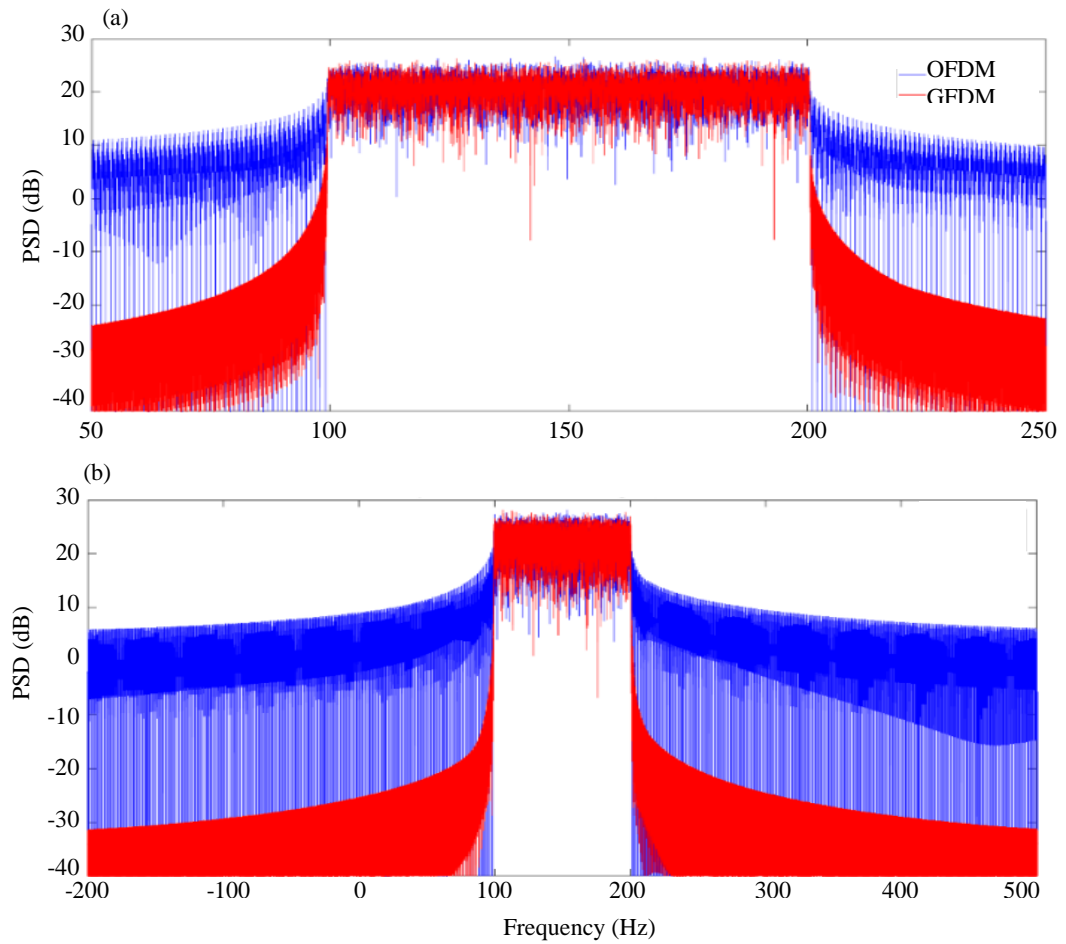

Fig. 6: Spectra of the OFDM and GFDM signals obtained from our simulations: a) Spectra obtained with 512 subcarriers and b) Spectra obtained with 1024 subcarriers 
Table 2: Input parameters for the GFDM system

\begin{tabular}{ll}
\hline Parameters & Value or types \\
\hline Length of IFFT/FFT & $\mathrm{N}=4096$ \\
Subsymbols (M) & $\mathrm{M}=15$ \\
Subcarriers (K) & $\mathrm{K}=512$ and $\mathrm{K}=1024$ \\
Pulse filter & OFDM: Rect. and GFDM: RRC, . =0.1 \\
Modulation & 16 OQAM \\
SNR & 15 and $20 \mathrm{~dB}$ \\
Bandwidth & $\mathrm{B}=9 \mathrm{MHz}$ \\
Period of symbol & $224 \mathrm{e}$ \\
Period of baseband & $1.0938 \mathrm{e}$ \\
Channel & AWGN \\
\hline
\end{tabular}

\section{CONCLUSION}

In this study, we conducted a comprehensive evaluation of 10 technologies that are expected to improve or change future $5 \mathrm{G}$ communication structures significantly. The requirements of $5 \mathrm{G}$ mobile networks were explained and the ways in which the above-mentioned technologies can facilitate the fulfilment of these requirements as well as their expected applications were described. According to, our simulation results, the spectra obtained using the GFDM technique might be better suited to $5 \mathrm{G}$ networks than those obtained using the OFDM technique. In addition, the use of GFDM leads to SER reduction owing to its design. This simulation-based evaluation was primarily performed to predict and counter the drawbacks of OFDM structures. By decreasing the CP value and broadcasting information in the form of a block, the transmission-time delay can be minimized, thereby improving synchronization and latency. In addition, this process could improve the spectral efficiency and allow the integration of MIMO technology. All of these factors are critical upgrades that align well with the predicted requirements of $5 \mathrm{G}$ structures. Furthermore, the reduced IFI, ICI, SER, OOB, PAPR and ISI will facilitate better utilization of the available channel capacity, thereby increasing data rates.

\section{ACKNOWLEDEGMENT}

The researcher would like to thank the University of Alkafeel for financial support of this project.

\section{REFERENCES}

Akyildiz, I.F., A. Lee, P. Wang, M. Luo and W. Chou, 2014a. A roadmap for traffic engineering in SDN-OpenFlow networks. Comput. Networks, 71: $1-30$.

Akyildiz, I.F., D.M. Gutierrez-Estevez, R. Balakrishnan and E. Chavarria-Reyes, 2014b. LTE-Advanced and the evolution to Beyond 4G (B4G) systems. Phys. Commun., 10: 31-60.
Akyildiz, I.F., M. Pierobon, S. Balasubramaniam and Y. Koucheryavy, 2015b. The internet of bio-nano things. IEEE. Commun. Mag., 53: 32-40.

Akyildiz, I.F., P. Wang and S.C. Lin, 2015a. SoftAir: A software defined networking architecture for $5 \mathrm{G}$ wireless systems. Comput. Networks, 85: 1-18.

Akyildiz, I.F., S. Nie, S.C. Lin and M. Chandrasekaran, 2016. 5G roadmap: 10 key enabling technologies. Comput. Networks, 106: 17-48.

Akyildiz, I.F., S.C. Lin and P. Wang, 2015c. Wireless software-defined networks (W-SDNs) and Network Function Virtualization (NFV) for $5 \mathrm{G}$ cellular systems: An overview and qualitative evaluation. Comput. Networks, 93: 66-79.

Al-Fuqaha, A., M. Guizani, M. Mohammadi, M. Aledhari and M. Ayyash, 2015. Internet of things: A survey on enabling technologies, protocols and applications. IEEE. Commun. Surv. Tutorials, 17: 2347-2376.

Alves, H., R.D. Souza and M.E. Pellenz, 2015. Brief survey on full-duplex relaying and its applications on $5 \mathrm{G}$. Proceedings of the 2015 IEEE 20th International Workshop on Computer Aided Modelling and Design of Communication Links and Networks (CAMAD), September 7-9, 2015, IEEE, Guildford, UK., pp: 17-21.

Andrews, J.G., S. Buzzi, W. Choi, S.V. Hanly and A. Lozano et al., 2014. What will 5G be? IEEE. J. Sel. Areas Commun., 32: 1065-1082.

Anonymous, 2010. 1901-2010-IEEE standard for broadband over power line networks: Medium access control and physical layer specifications. IEEE, New York, USA. http://ieeexplore.ieee.org/ document/5678772/.

Anonymous, 2014a. DOCOMO 5G white paper, 5G radio access: Requirements, concept and technologies. NTT DoCoMo, Chiyoda, Tokyo, Japan.

Anonymous, 2014b. In the matter of use of spectrum bands above $24 \mathrm{GHz}$ for mobile radio services. Federal Communications Commission, Washington, DC., USA.

Anonymous, 2014c. SK telecom 5G white paper: SK telecom's view on 5G vision, architecture, technology and spectrum. SK Telecom, Jung District, Seoul, South Korea.

Anonymous, 2015a. IMT vision-Framework and overall objectives of the future development of IMT for 2020 and beyond. ITU Radiocommunication Sector, Geneva, Switzerland. http:/www.itu.int dms_pubrec/itu-r/rec/m/R-REC-M.2083-0-201509I!!PDF-E.pdf 
Anonymous, 2015b. Notice of proposed rulemaking, use of spectrum bands above $24 \mathrm{GHz}$ for mobile radio services. Federal Communications Commission, Washington, DC., USA.

Anonymous, 2015c. Project FP 2012-2015. Cordis, Hong Kong, Shanghai Street, Mong Kok, Hong Kong. https://cordis.europa.eu/guidance/archive en.html

Anonymous, 2016. Evolved Universal Terrestrial Radio Access (E-UTRA) and evolved universal terrestrial radio access network (E-UTRAN): Overall description Stage 2. 3GPP Mobile Competence Centre, Valbonne, France.

Anonymous, 2016b. ProgRAN. Argela, Maslak, Turkey, Sarýyer. https://www.argela.com.tr/progran/

Asadi, A., Q. Wang and V. Mancuso, 2014. A survey on device-to-device communication in cellular networks. IEEE. Commun. Surv. Tutorials, 16: 1801-1819.

Banelli, P., S. Buzzi, G. Colavolpe, A. Modenini and F. Rusek et al., 2014. Modulation formats and waveforms for $5 \mathrm{G}$ networks: Who will be the heir of OFDM?: An overview of alternative modulation schemes for improved spectral efficiency. IEEE. Signal Proc. Mag., 31: 80-93.

Bangerter, B., S. Talwar, R. Arefi and K. Stewart, 2014. Networks and devices for the $5 \mathrm{G}$ era. IEEE. Commun. Mag., 52: 90-96.

Bansal, M., J. Mehlman, S. Katti and P. Levis, 2012. Openradio: A programmable wireless dataplane. Proceedings of the 1st International Workshop on Hot Topics in Software Defined Networks, August 13, 2012, ACM, Helsinki, Finland, ISBN:978-14503-1477-0, pp: 109-114.

Boccardi, F., W. Heath Jr., A. Lozano, T.L. Marzetta and P. Popovski, 2014. Five disruptive technology directions for 5G. IEEE. Commun. Mag., 52: 74-80.

Cisco, 2015. Cisco visual networking index: Forecast and methodology. Cisco, San Jose, California, USA.

Dely, P., J. V estin, A. Kassler, N. Bayer and H. Einsiedler et al., 2012. CloudMAC-An openflow based architecture for 802.11 MAC layer processing in the cloud. Proceedings of the 2012 IEEE International Workshops on Globecom (GC Wkshps), December 3-7, 2012, IEEE, Anaheim, California, USA., ISBN:978-1-4673-4942-0, pp: 186-191.

Di Taranto, R., L.S. Muppirisetty, R. Raulefs, D. Slock and T. Svensson et al., 2014. Location-aware communications for $5 \mathrm{G}$ networks: How location information can improve scalability, latency and robustness of 5G. IEEE. Signal Proc. Mag., 31: 102112.
Duan, X. and X. Wang, 2015. Authentication handover and privacy protection in $5 \mathrm{G}$ hetnets using software-defined networking. IEEE. Commun. Mag., 53: 28-35.

Farhang-Boroujeny, B., 2011. OFDM versus filter bank multicarrier. IEEE. Signal Proc. Mag., 28: 92-112.

Fettweis, G.P., 2014. The tactile internet: Applications and challenges. IEEE. Veh. Technol. Magazine, 9: 64-70.

Fodor, G., E. Dahlman, G. Mildh, S. Parkvall and N. Reider et al., 2012. Design aspects of network assisted device-to-device communications. IEEE. Commun. Mag., 50: 170-177.

Fodor, G., S. Parkvall, S. Sorrentino, P. Wallentin and Q. Lu et al., 2014. Device-to-device communications for national security and public safety. IEEE. Access, 2: $1510-1520$.

Ghosh, A., T.A. Thomas, M.C. Cudak, R. Ratasuk and P. Moorut et al., 2014. Millimeter-wave enhanced local area systems: A high-data-rate approach for future wireless networks. IEEE J. Selected Areas Commun., 32: 1152-1163.

Gudipati, A., D. Perry, L.E. Li and S. Katti, 2013. SoftRAN: Software defined radio access network. Proceedings of the 2nd International ACM SIGCOMM Workshop on Hot Topics in Software Defined Networking, August 16, 2013, ACM, Hong Kong, China, ISBN:978-1-4503-2178-5, pp: 25-30.

Hossain, E. and M. Hasan, 2015. 5G cellular: Key enabling technologies and research challenges. IEEE. Instrum. Meas. Mag., 18: 11-21.

Jain, S., A. Kumar, S. Mandal, J. Ong and L. Poutievski et al., 2013. B4: Experience with a globally-deployed software defined WAN. Proceedings of the 2013 International Conference on ACM SIGCOMM, August 12-16, 2013, ACM, Hong Kong, China, ISBN:978-1-4503-2056-6, pp: 3-14.

Jin, X., L.E. Li, L. Vanbever and J. Rexford, 2013. Softcell: Scalable and flexible cellular core network architecture. Proceedings of the ACM 9th International Conference on Emerging Networking Experiments and Technologies, December 9-12, 2013, ACM, Santa Barbara, California, USA., ISBN:978-1 4503-2101-3, pp: 163-174.

Kaewpuang, R., D. Niyato, P. Wang and E. Hossain, 2013. A framework for cooperative resource management in mobile cloud computing. IEEE J. Selected Areas Commun., 31: 2685-2700.

KaiTen, F., H. Chung Hsien and L. TseEn, 2008. Velocity-Assisted Predictive Mobility and Location-Aware Routing Protocols for Mobile Ad Hoc Networks. IEEE Trans. VEH. Technol., 57: 448-464. 
Larsson, E.G., O . Edfors, F. Tufvesson and T.L. Marzetta, 2014. Massive MIMO for next generation wireless s ystems. IEEE. Commun. Mag., 52: 186-195.

Lin, X., J. Andrews, A. Ghosh and R. Ratasuk, 2014. An overview of 3GPP device-to-device proximity services. IEEE. Commun. Mag., 52: 40-48.

Lin, Y.D. and Y.C. Hsu, 2000. Multihop cellular: A new architecture for wireless communications. Proceedings of the IEEE 19th Annual Joint Conference of the IEEE Computer and Communications Societies, Volume 3, March 26-30, 2000, Tel Aviv, Israel, pp: 1273-1282.

Mahmood, N.H., G. Berardinelli, F.M. Tavares and P.E. Mogensen, 2015. On the potential of full duplex communication in $5 \mathrm{G}$ small cell networks. Proceedings of the IEEE 2015 81st International Conference on Vehicular Technology (VTC Spring), May 11-14, 2015, IEEE, Glasgow, UK., pp: 1-5.

Marzetta, T.L., 2010. Noncooperative cellular wireless with unlimited numbers of base station antennas. IEEE. Trans. Wirel. Commun., 9: 3590-3600.

Michailow, N., M. Matthe, I.S. Gaspar, A.N. Caldevilla and L.L. Mendes et al., 2014. Generalized frequency division multiplexing for 5 th generation cellular networks. IEEE. Trans. Commun., 62: 3045-3061.

Morelli, M., C.C.J. Kuo and M.O. Pun, 2007. Synchronization techniques for orthogonal frequency division multiple access (OFDMA): A tutorial review. Proc. IEEE., 95: 1394-1427.

Osseiran, A., F. Boccardi, V. Braun, K. Kusume and P. Marsch et al., 2014. Scenarios for 5G mobile and wireless communications: The vision of the METIS project. IEEE. Commun. Mag., 52: 26-35.

Ramadhan, A.J., 2015. A novel MIMO-OFDM technique for improving wireless communications system performance based on SF-BC. Intl. J. Comput. Appl., 131: 28-31.

Ramadhan, A.J., 2017. Implementation of 5G FBMC PHYDYAS prototype filter. Intl. J. Appl. Eng. Res., 12: 13476-13481.

Rodriguez, J., 2015. Fundamentals of 5G Mobile Networks. John Wiley \& Sons, Hoboken, New Jersey, USA., ISBN:9781118867471, Pages: 336.

Rusek, F., D. Persson, B.K. Lau, E.G. Larsson and T.L. Marzetta et al., 2013. Scaling up MIMO: Opportunities and challenges with very large arrays. IEEE. Signal Process. Mag., 30: 40-60.
Schaich, F. a nd T. Wild, 2014. Wave form contenders for $5 \mathrm{G}-\mathrm{OFDM}$ vs. FBMC vs. UFMC. Proceedings of the 6th International S y mposium on Communications, Control and Signal Processing (ISCCSP'14), May 21-23, 2014, IEEE, Athens, Greece, I SBN:978-1-47992890-3, pp: 457-460.

Schaich, F., T. Wild and Y. Chen, 2014. Waveform contenders for $5 \mathrm{G}$-suitability for short packet and low latency transmissions. Proceedings of the 2014 IEEE 79th International Conference on Vehicular Technology (VTC Spring), May 18-21, 2014, IEEE, Seoul, South Korea, pp: 1-5.

Shariatmadari, H., R. Ratasuk, S. Iraji, A. Laya and T. Taleb et al., 2015. Machine-type communications: Current status and future perspectives toward $5 \mathrm{G}$ systems. IEEE. Commun. Mag., 53: 10-17.

Suresh, L., J. Schulz-Zander, R. Merz, A. Feldmann and T. Vazao, 2012. Towards programmable enterprise WLANS with Odin. Proceedings of the 1st International Workshop on Hot Topics in Software Defined Networks (HotSDN '12), August 13, 2012, ACM, New York, USA., ISBN:978-1-4503-1477-0,pp: 115-120.

Tehrani, M.N., M. Uysal and H. Yanikomeroglu, 2014. Device-to-device communication in 5G cellular networks: Challenges, solutions and future directions. IEEE. Commun. Mag., 52: 86-92.

Van den Abeele, F., J. Hoebeke, G.K. Teklemariam, I. Moerman and P. Demeester, 2015. Sensor function virtualization to support distributed intelligence in the internet of things. Wireless Pers. Commun., 81: 1415-1436.

Vermesan, O. and P. Friess, 2013. Internet of Things: Converging Technologies for Smart Environments and Integrated Ecosystems. Rivers Publisher, New York USA., ISBN:978-87-93102-94-1, Pages: 351.

$\mathrm{Wu}$, J., Z. Zhang, Y. Hong and Y. Wen, 2015. Cloud radio access network (C-RAN): A primer. IEEE. Netw., 29: $35-41$.

Yap, K.K., R. Sherwood, M. Kobayashi, T.Y. Huang and M. Chan et al., 2010. Blueprint for introducing innovation into wireless mobile networks. Proceedings of the 2nd ACM SIGCOMM Workshop on Virtualized Infrastructure Systems and Architectures (VISA '10), September 3, 2010, ACM, New York, USA., ISBN:978-1-4503-0199-2, pp: 25-32.

Zhang, X., W. Cheng and H. Zhang, 2015. Full-duplex transmission in $\mathrm{PHY}$ and MAC layers for $5 \mathrm{G}$ mobile wireless networks. IEEE. Wireless Commun., 22: 112-121. 